\title{
The Final Size of the Universe Based on the Elasticity of the Fabric of Spacetime
}

\author{
Geo Douglas $^{1 *}$, Naman S. Bajaj ${ }^{2}$ \\ ${ }^{1}$ Author and Independent Researcher, New Jersey, USA \\ ${ }^{2}$ College of Engineering, Pune, India \\ Email: ${ }^{\star}$ Geo@GeoDouglas.com
}

How to cite this paper: Douglas, G. and Bajaj, N.S. (2020) The Final Size of the Universe Based on the Elasticity of the Fabric of Spacetime. Journal of High Energy Physics, Gravitation and Cosmology, 6, 450-461.

https://doi.org/10.4236/jhepgc.2020.63035

Received: May 27, 2020

Accepted: July 25, 2020

Published: July 28, 2020

Copyright $\odot 2020$ by author(s) and Scientific Research Publishing Inc. This work is licensed under the Creative Commons Attribution International License (CC BY 4.0).

http://creativecommons.org/licenses/by/4.0/

\begin{abstract}
We investigate the fabric of spacetime, its ability to stretch, curve, and expand. Through our continuous studies of accretion disks located at the core of galaxies, it is our conclusion that these disks are separate from the host galaxy stellar disk. Our research has also determined that the radius of accretion disks in spiral galaxies follow a consistent ratio according to the circumference of their adjacent supermassive black hole based on its Schwarzchild radius. We present evidence suggesting that galactic accretion disks are a key element to understand galaxy formation and can provide a precise calculation to how much the fabric of space will stretch. Once the degree of the elasticity of spacetime was established, we applied these measurements to the size of the universe at 380,000 years of age based on the imagery of the cosmic microwave background. This calculation provided us with the maximum diameter the universe will reach, an exact time when the universe will stop expanding, and where we are today within that timeline.
\end{abstract}

\section{Keywords}

Accretion Disk, Supermassive Black Hole, Galaxy Formation, Universe, Fabric of Space

\section{Introduction}

How did the Universe come into existence? How did it evolve from a state of chaos to an expanding and organized entity with over one hundred billion galaxies? These are the biggest questions of cosmology and still remain inconclusive to a certain extent.

In the $20^{\text {th }}$ Century, the steady-state model of the universe and the big bang scenario were two major contenders for explaining the past, present and future of the universe (Gruenbaum et al. 1989) [1]. In the 1950s, many evidences were 
found to contradict the steady-state model, and the majority of support began swaying toward the big bang. Among the evidence was the imagery of the cosmic microwave background and the Hubble-Lemaitre law that greatly supports the expansion of the universe (Smoot et al. 1991, Sandage et al. 1989) [2] [3].

Of course, we must consider Albert Einstein's theory of general relativity which successfully explains all the major interactions within the universe. General relativity has been tested repeatedly and continues to be proven correct. Currently, the field equations of general relativity are determined to be the mathematical foundation of the universe, especially at a macro-level (Lorentz et al. 1952) [4].

However, at the same time, Max Planck was orchestrating his own equations and put forth quantum theory, which also has been proven correct repeatedly throughout the years and is the foundation of the universe on a micro-level (Klein et al. 1961) [5].

Since that time, a major problem in science developed as the mathematics of general relativity and quantum theory do not sync together. In order to attempt to bring the foundations of the micro (quantum theory) together with the foundations of the macro (general relativity), two other theories surfaced, quantum loop gravity and string theory. Both quantum loop gravity and string theory provide different descriptions of spacetime properties and how the macro and micro link together (Rovelli et al. 2008, Luest et al. 1989) [6] [7].

More recently, in 2003, another piece of the unsolved puzzle came on the scene in regard to how large the universe would become before it potentially destroys itself. This is referred to as the big rip. This concept originated from the observation that the expansion rate of the universe has been increasing with time since the universe was 7.5 billion years old (Hubble et al. 1929) [8]. According to this theory, after a finite amount of time, all atoms and the cosmological structure of spacetime itself would rip apart to mark the end of the universe (Caldwell et al. 2003) [9].

Here, through this paper, we present a model that could be considered a hybrid of the aforementioned suggesting the ultimate size of the universe and the time in which it will be achieved. Whether or not the big rip would occur at the time noted in this paper is undetermined. If the universe does not rip apart at this point in time or prior, perhaps, the process of the big crunch may begin, a time where the universe stops expanding and begins compressing back on itself as the gravity from all the mass in the universe would begin to pull back on the outer boundaries. It is also plausible to consider that nothing would happen at the time of maximum expansion and the universe would become a static vacuum. In other terms, the universe would include the same properties, however without significant expansion.

Nonetheless, this theory has many different moving parts and factors to consider. In order to present this theory in an organized fashion, it must be broken down into 3 different sections. 
1) Accretion disk and supermassive black holes size ratio.

2) Accretions disk and supermassive black hole creation.

3) Determining the maximum size of the universe.

\section{Accretion Disk and Supermassive Black Holes Size Ratio}

The word accretion is the accumulation of particles in the form of gasses and matter which are gravitationally attracted into a flat, symmetrical disk-like formation surrounding an object of superior mass. Through observations right in our own solar system, we can observe accretion effects through the rings of Saturn, the orbital patterns of moons in planetary systems, and ultimately why most of the planets in our solar system rotate around the sun in a flat disk-like fashion and in the same direction.

Spiral galaxies as a whole, are also a form of accretion. The disk-like shape of the entire galaxy is spinning around its most centric dominating force of gravity, its supermassive black hole. However, physics has determined that there may be other forces that play a role in keeping galaxies intact. Many authorities in the scientific community attribute this to dark matter, while still maintaining accordance with general relativity (Trimble et al. 1987, Corda et al. 2009) [10] [11].

\subsection{Schwarzschild Radius}

In the theory of general relativity by Albert Einstein, he added ten field equations that proposed the fundamentals of gravity and its effect on spacetime curvature due to mass and energy [4].

In 1916, Karl Schwarzschild, formulated a spherical, symmetric solution to Einstein's field equations that provides the precise radius of the event horizon surrounding a black hole. Through utilizing his equation, we can determine if any object of mass is classified as an actual black hole if its physical radius is smaller than its Schwarzschild radius (Rs). The Rs also points directly to where the singularity of the black hole would be located.

$$
r_{s}=\frac{2 G M}{c^{2}}
$$

\subsection{Presented Ratio}

Through our research specifically on the relationship between accretion disks and their adjacent supermassive black hole (SMBH), we present a consistent ratio based on the radius of accretion disks according to the circumference of the $\mathrm{SMBH}$. The equation we use to determine the circumference of a SMBH is by multiplying 2 times $\pi$, times the Schwarzschild Radius of the SMBH: $\mathrm{C}=2 \pi \mathrm{Rs}$.

Through our search for consistent and accurate accretion disk data, (Kuo et al. 2010) [12] feature seven active, spiral quasars, NGC 1194, NGC 2273, UGC 3789, NGC 2960, NGC 4388, NGC 6264, and NGC 6323. Out of these seven examples, five of them fall within $98.3 \%$ accuracy and average at a ratio of 26.7 
as shown in Figure 1. We obtain this ratio by the accretion disk radius divided by the circumference of its supermassive black hole.

Galaxies that do not follow this specific ratio, are not categorized as active or spiral, or may have unique characteristics. The two quasars listed in the [12] that did not fall within this range were still considered active, spiral galaxies, however they are two unique cases.

NGC 2273 is a large galaxy spanning approximately 100,000 light years in diameter (close to the size of the Milky Way) and is located in the Lynx constellation. This galaxy is approximately 95 million light years from earth and is considered to

Table 1. As defined in the chart above, these disk radius to SMBH circumference ratios are consistent and suggest a unique relationship between them. From the smallest SMBH to the largest, over three times the size, the ratio remains consistent with a very small margin of error.

\begin{tabular}{|c|c|c|c|c|c|c|}
\hline $\begin{array}{c}\text { Black Hole/ } \\
\text { Quasar }\end{array}$ & $\begin{array}{c}\text { Distance from } \\
\text { Earth (Ly) }\end{array}$ & $\begin{array}{c}\text { Mass } \\
\left({ }^{*} 10^{7} \mathrm{M}(\text { sun })\right)\end{array}$ & $\begin{array}{c}\text { Schwarzschild Radius } \\
\qquad\left({ }^{*} 10^{10} \mathrm{~km}\right)\end{array}$ & Circumference $(C)\left({ }^{*} 10^{10} \mathrm{~km}\right)$ & $\begin{array}{l}\text { Disk Outer Radius } \\
\text { (R) }\left({ }^{*} 10^{13} \mathrm{~km}\right)\end{array}$ & Ratio (R/C) \\
\hline UGC 3789 & $1.63 \mathrm{E}+08$ & $1.12 \pm 0.05$ & $3.3085273 \pm 0.14770211$ & $20.78809012 \pm 0.9280397274$ & 2.0055 & $22.756-24.89$ \\
\hline NGC 2960 & $2.32 \mathrm{E}+08$ & $1.14 \pm 0.05$ & $3.367608 \pm 0.14770211$ & $21.15930511 \pm 0.9280397274$ & 0.49371 & $25.14-27.41$ \\
\hline NGC 4388 & $6.20 \mathrm{E}+07$ & $0.84 \pm 0.02$ & $2.481395 \pm 0.0590808$ & $15.59106461 \pm 0.3712156145$ & 0.5554 & $28.033-29.363$ \\
\hline NGC 6264 & $4.44 \mathrm{E}+08$ & $2.84 \pm 0.04$ & $8.38948 \pm 0.1181617$ & $52.71265747 \pm 0.7424318573$ & 1.2034 & $22.51-23.16$ \\
\hline NGC 6323 & $3.43 \mathrm{E}+08$ & $0.93 \pm 0.01$ & $2.747259 \pm 0.02954042$ & $17.26153738 \pm 0.1856079329$ & 0.46285 & $26.54-27.11$ \\
\hline
\end{tabular}
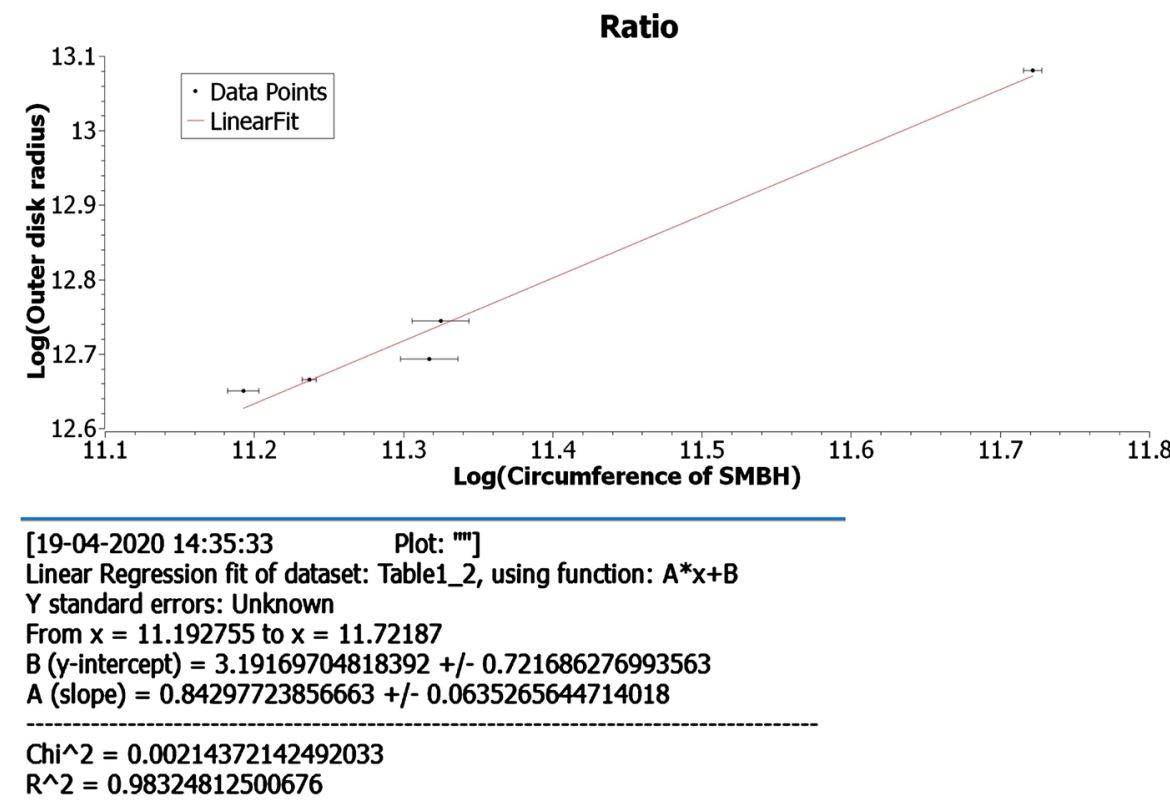

Figure 1. Represents the variation of the outer accretion disk radius with respect to the Rs to determine the circumference of the SMBH. Each dot represents an entry from Table 1. The black lines are the error bars on the circumference of the SMBH which originate from the error in calculating the mass of these SMBHs. The red line is a linear curve fit on data points. The accuracy with which we have a fit is approximately $98.3 \%$ which shows that the observed data is in good agreement with the posed equation. The remaining error can be attributed to the inefficiency in calculating the outer disk radius (which here is considered 0 ). 
be a ring galaxy. It features an inner ring and two outer pseudo-rings. These unique features are powered by two spiral arms that contain large amounts of molecular gas that may indicate active star formation. Due to this unique structure, this galaxy contains a very small accretion disk in comparison to the mass of its supermassive black hole as its ratio came in at only 9.805 (Erwin and Sparke, 2003) [13].

NGC 1194 came in at a ratio of 17.707 , however we are getting fluxuations in measurements. As mentioned in this paper, (Greene et al. 2014) [14] accurate measurements are difficult to obtain on this particular galaxy. As we look closer at NGC 1194, we are confident the ratio will increase accordingly.

\section{Accretions Disk and Supermassive Black Hole Creation}

First, to avoid confusion, we must consider that most large objects with superior mass throughout spacetime have an accretion effect. Even in cases where there are minimal amounts of swirling gasses or matter around an object, it is still categorized as an actual accretion disk. Stellar-mass black holes also have the capacity to evoke an accretion process with gases and matter. However, in both cases, it is a much different process than what occurs at the core of galaxies. (Gilfanov et al. 2015) [15]. Just as there are two major types of black holes, stellar-mass and supermassive, and two types of singularities, gravitational and ring singularities, it is our suggestion for two different categories of accretion disks. We have taken this step within this paper and have referred to accretion disks that are located at the core of galaxies as galactic accretion disks (GAD).

\subsection{Supermassive Black Hole Creation}

Albert Einstein's theory of general relativity is deeply rooted in Riemannian geometry which proposes that spacetime can ripple, dent, and bend, but must remain intact, unless to a point of a singularity. However, string theory supports the possibilities of tears or rips within the fabric of space, claiming it is attainable and compensated for mathematically, without breaking any laws of physics. However, the scientific community continues to find evidence which would make it quite impossible to conclude supermassive black holes are formed by a collapsing star. The technology of the Magellan telescopes in Chile and the (WISE) Wide-field Infrared Survey Explorer, has revealed the most distant, young black holes with a redshift of $7+$ and now even $8+$ prompting an excess of 13 billion years in age (Bouwens et al. 2004, Bouwens et al. 2011, Hayes et al. 2012) [16] [17] [18]. In some cases, it only leaves 600,000 years to work with. How did massive quasars and entire galaxies form within 600,000 years?

(Basu et al. 2019) [19] in the paper titled, the Mass Function of Supermassive Black Holes in the Direct-collapse Scenario, published in 2019, is one example that conclusively indicates that Quasar J1342 + 0928 could not be related to a direct-collapse scenario, as it could not have the time to complete the super-Eddington accretion process. We can conclude that stellar-mass black holes 
are created by a direct-collapse, but in the case of SMBHs, searching for alternatives is an essential component to expanding our knowledge of cosmology.

Through analyzing the cosmography work of Hélène Courtois and her team at the University of Lyon in France, there is an apparent cosmic flow (Pomarede et al. 2017) [20]. By observing the presented patterns of nature, and understanding that superclusters are essentially cosmic basins, as quoted by Hélène Courtois, we can conclude that cosmic flow is a major part of our universe.

Additionally, when more distant galaxies are separating at speeds that exceed the speed of light, it's apparent that massive sections of spacetime, multiple megaparsecs, are shifting in every direction at high speeds. Combining that fact with the majority of our universe (68\%) consisting of dark energy (Amendola et al. 2003) [21], deemed responsible for the explanation of the universe, it's plausible to anticipate unmeasured forces contribute to excessive momentum and velocity that would certainly place an enormous amount of pressure on the fabric of space in vulnerable regions throughout the universe. Dark matter, $(27 \%$ of the universe) also deemed responsible for keeping galaxies intact, would also account for more pressure and force against the four-dimensional fabric of spacetime (Hui et al. 2017) [22].

Furthermore, it is important to understand the definition of a singularity according to general relativity. We quote B. G. Schmidt's definition from 1971, "In the internal geometry all time-like radial geodesics of the collapsing star terminate after a lapse of finite proper time in the termination point $r=0$ and it is impossible to extend the internal space-time manifold beyond that termination point that is called singularity point" (Schmidt et al. 1971) [23], however this only occurs in non-rotating black holes. Considering this paper focuses on spiral galaxies and their SMBHs, these are in fact rotating, which therefore did not form a gravitational singularity, instead they acquired ring singularity (or ringularity). Ring singularities follow the Kerr metric, a solution that was also derived from Albert Einstein's field equations. These ring singularities encompass a much larger area at the center of black holes compared to gravitational singularities.

As cited by Stanford University in 2019 (Curiel et al. 2019) [24], the scientific community accepts a spacetime tear responsible for singularities. The debate and often misconception lays within the size of the tear. As previously mentioned, if a supermassive black hole started from a direct collapse scenario the process to become the behemoth galaxy such as Quasar J1342 + 0928 (Banados et al. 2018) [25], would not have the time to complete the super-Eddington accretion process considering the age of the universe at the time of observation. To account for the size of these SMBHs, other scientists have raised the possibility of multiple mergers of stellar-mass black holes, however considering there are only 600,000 years to work with, it is also problematic to suggest that stars not only had the time to form, and to eventually collapse into stellar-mass black holes, but also the time to merge with adjacent objects which took the same course of action. Due to this improbability, these ancient SMBHs may have been created by 
a tear of a more significant spatial region.

\subsection{Galactic Accretion Disk Creation}

There are many examples discovered within the last decade of GADs often spinning faster than expected. When we measure disks spinning close to the speed of light (Volonteri et al. 2015) [26], it provides clues to what actually is occurring at the most centric location of galaxies. There are also examples of accretion disks rotating in the opposite direction to the SMBH. This Counter-rotating effect adds evidence that GADs do not follow typical accretion processes as other examples outside galaxy centers (Kuznetsov et al. 1999, Shapiro et al. 1976) [27] [28]. It is also evident that the disk itself is not always coplanar to the host galaxy stellar disk, meaning the galaxy as a whole. These angular variations suggest that the GAD is a separate entity layered over the top of the host galaxy stellar disk as suggested in the following two papers published by Cornell University on arxiv (Kaviraj et al. 2015, Middleton et al. 2016) [29] [30].

Factoring the excessive speeds, examples of counter-rotation and the angular variations of the accretion disk compared to the host galaxy stellar disk, and especially considering the ratio that is maintained between the supermassive back hole and its accretion disk, it is our determination that the GAD are separate objects with their own unique properties, and an actual remnant leftover from the initial tear in the fabric of spacetime that created the SMBH.

That being said, the symmetrical remnant from the spacetime tear is an actual layer of the fabric of space. As it separated and became its own object, it began spinning and expanding, eventually to become the accretion disk covering a much larger area compared to the circumference of the $\mathrm{SMBH}$. This would account for the relationship and their consistent size ratio.

The fact that spacetime is transparent, the gasses and matter on the disk would naturally push outward just as all accretion processes and examples such as a solar system. The inner disk diameter is simply where the hot temperature gasses begin on the disk. This is usually determined by the rotation speed of the $\mathrm{SMBH}$ and follows the Newtonian mechanics $\mathrm{r}$ ISCO (innermost stable circular orbit radius). The faster the black hole spins, the smaller the radius becomes of
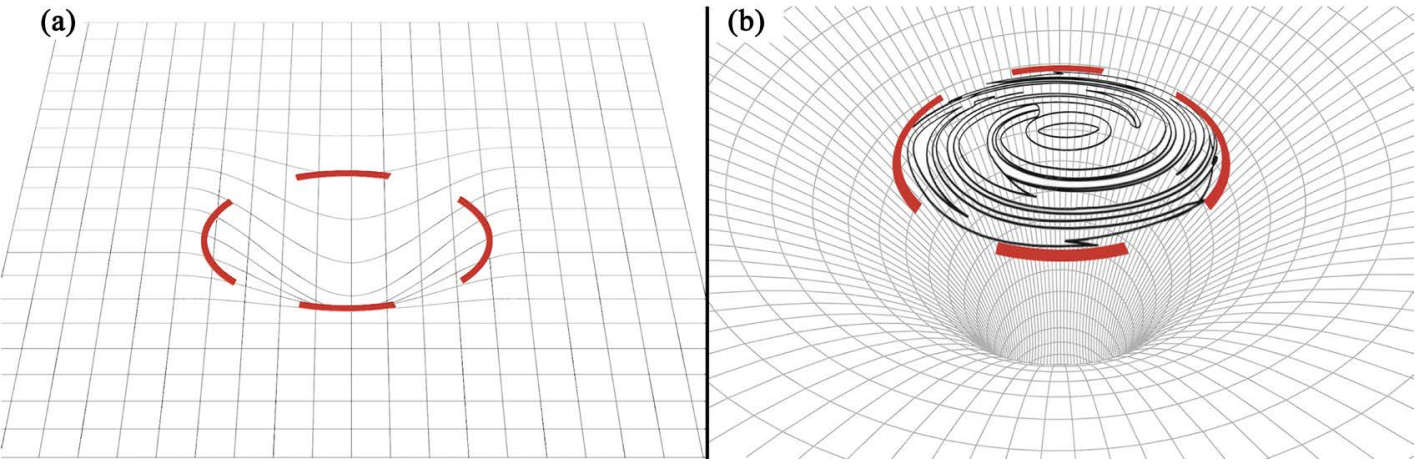

Figure 2. The image on the left illustrates the initial tear to form the SMBH. Figure 2(b) presents the leftover remnant from the initial tear of Figure 2(a). 
the inner accretion disk in regard to where gas and matter are observed.

The remaining inner part of the GAD would be a transparent layer over the SMBH itself, which may account for how active galactic nuclei (AGN) would get trapped underneath this layer of spacetime and build up to incredible measures of energy and luminosity, resulting in the formation of a quasar.

\subsection{Determining Tear Size}

Considering the relationship between the accretion disk and its supermassive black hole as presented in Figure 2, and the consistent radius to circumference ratio averaging 26.7, we maintained this exact formula to determine the size of the spacetime tear that created the SMBH. Again, the equation used to obtain the 26.7 ratio derives from the radius of the accretion disk to the circumference of the SMBH $\mathrm{R}=\mathrm{C}$ (26.7). We use the same ratio and equation applied to the size of the initial tear, Rs $=\mathrm{C}$ (26.7) In this case, the circumference in this equation represents the tear size that created the SMBH. This equation is also in direct correlation with the Kerr metric that considers mass and angular momentum.

Using this equation, we have calculated an average spacetime tear size between UGC 3789, NGC 2960, NGC 4388 and NGC 6323 which equated to $1.141507 \mathrm{e}+9 \mathrm{Km}$ in circumference which would have a tear radius just over one astronomical unit. (Although the ratio is the same, due to the much larger size of NGC 6264, it is excluded in this example for consistency purposes).

The average GAD radius of UGC 3789, NGC 2960, NGC 4388 and NGC 6323 amounts to $4.89845811 \mathrm{e}+12 \mathrm{Km}$. Through using the same radius to circumference concept, we find an average from the tear circumference to the accretion disk radius as 4291.22 times greater. $\mathrm{R}=\mathrm{C}$ (4291.33). In this equation, $\mathrm{R}$ represents the average GAD radius. $C$ represents the average tear circumference. This critical equation suggests the degree of the elasticity of spacetime.

\section{Determining the Size of the Universe}

In order to compare the degree of elasticity against the universe as a whole, we must have a starting point. Fortunately, the most significant image in cosmology, the cosmic microwave background (CMB) has specific temperatures, size and age of the universe at the point in time of the imagery.

The CMB photons initially had an estimated temperature of 3000 kelvin. Now estimates are more accurate at a temperature of 2,725 kelvin which indicates a redshift of 1100 as shown in Figure 3. According to NASA's reionization redshift model, the $\mathrm{CMB}$ would be 1100 times smaller than the size of the universe as we know it today (Kinney et al. 2003) [31].

Based on current estimates, the radius of the universe is now at 46.5 billion light-years. The estimated size of the universe at the time of the CMB when the universe was approximately 380,000 years old has been determined to be $84,545,454$ light-years in diameter. 


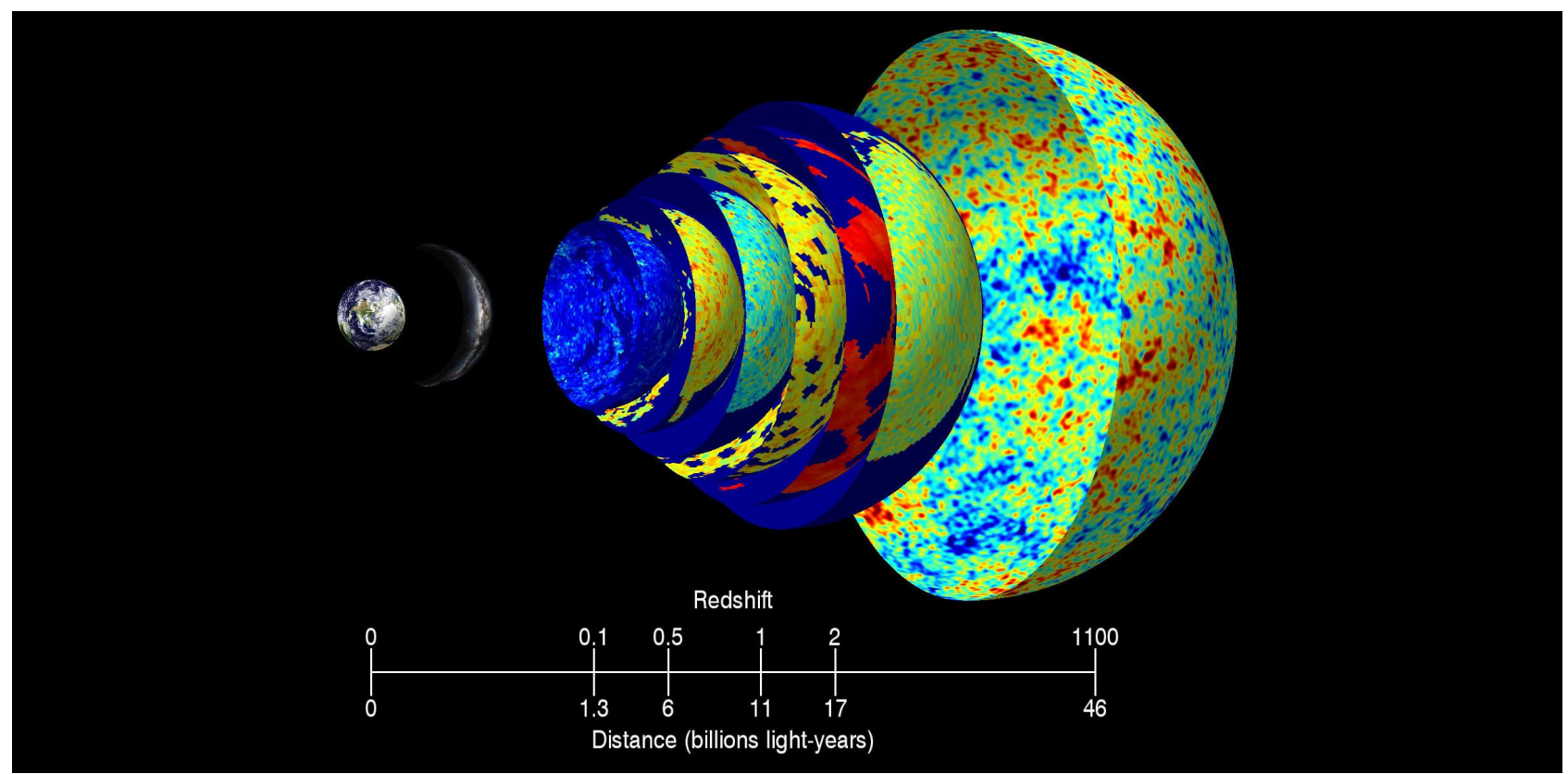

Figure 3. Image credit: Earth: NASA/Blue Earth; Milky Way: ESO/S. Brunier; CMB: NASA/WMAP.

By calculating the size of the universe at 380,000 years old at a circumference of 265,607,377 light-years and factoring in that the fabric of space stretches 4291.22 times greater from circumference to radius, we have concluded the universe will stop expanding when it reaches 1,139,779,688,330 light-years in radius, ultimately reaching a diameter of over two trillion light-years at precisely 2,279,559,376,660 light-years.

As of today, estimates suggest the universe is 93 billion light-years in diameter (Seargent et al. 2015) [32]. This would suggest that the current size of the universe is only $4.07 \%$ of what it will become. Considering the universe is only 13.8 billion years old, our calculations would suggest that the age of the universe will be 339,066,339,066 when it ceases to expand and reaches its maximum size.

\section{Summary}

By analysing the activity at the center of galaxies, and by presenting evidence that defines the consistent ratio between accretion disks and their SMBHs, ultimately directs logic toward galaxy formation. We describe the proposed event as a spacetime tear that is consistent with ring-singularities, and are in direct correlation with the Kerr metric without changing the Rs whatsoever.

Listed are the steps taken:

1) Accretion disk radius to SMBH circumference ratio averages to be 26.7 .

2) Illustrated the difference between a $\mathrm{SMBH}$ and a stellar mass black hole.

3) The difference between GADs and a typical accretion process in the universe.

4) A larger tear in the fabric of space is responsible for SMBH and galaxy formation.

5) The remnant left over from that tear stretched out to be the galaxy's accre- 
tion disk.

6) The presented scenario would account for AGN being trapped, resulting in quasar creation.

7) Presented a precise tear size equation for SMBH creation.

8) Described the elasticity of the fabric of space according to the stretch ratio of GADs.

9) Noted the CMB and its size at the time of imagery, 380,000 years old.

10) Compared the elasticity to the $C M B$ to derive at the maximum size of the universe.

Possible additional insights and further investigation of subjects where our theory may emerge are as follows; To suggest accretion disks at the most centric region of galaxies are an actual fragment of spacetime has a degree of conjecture, however the observations and data noted in this paper, including the referenced, indicate an apparent difference between accretion disks and host galaxy stellar disks. That being said, new data and more precise imagery may assist in further identifying the behavior and activity at the core of galaxies to present a threedimensional model.

We also must consider why elliptical galaxies have inconsistent accretion disk to SMBH ratios. This is an ongoing study, however at this time, we presume that most active, spiral galaxies are younger than elliptical galaxies and consider that most elliptical galaxies may be the result of at least one merger between two spiral galaxies. In the early universe, mergers of two galaxies had a higher probability and were a more frequent occurrence when the universe was a much smaller entity with less distance between galaxies.

It is our objective to further calculate the age of the universe when this will occur, not based on percentage, but to factor the fluctuating expansion speeds of the universe that could alter the presented number.

In conclusion, we are also confident the presented hypothesis has crossed the threshold into a viable theory through precise calculations, observations and references. With further consideration and application, this theory may eventually provoke a new model of galaxy formation and to further expand on the process the universe took from a state of chaos and entropy at its infancy to ultimately an organized, mathematical universe of today.

\section{Conflicts of Interest}

The authors declare no conflicts of interest regarding the publication of this paper.

\section{References}

[1] Grünbaum, A. (1989) The Pseudo-Problem of Creation in Physical Cosmology. Philosophy of Science, 56, 373-394. https://doi.org/10.1086/289497

[2] Smoot, G.F., Bennett, C.L., Kogut, A., Aymon, J., Backus, C., De Amici, G., et al. (1991) Preliminary Results from the COBE Differential Microwave Radiometers-Large Angular Scale Isotropy of the Cosmic Microwave Background. The As- 
trophysical Journal, 371, L1-L5. https://doi.org/10.1086/185988

[3] Sandage, A. (1989) Edwin Hubble 1889-1953. Journal of the Royal Astronomical Society of Canada, 83, 351-362.

[4] Lorentz, H.A., Einstein, A., Minkowski, H., Weyl, H. and Sommerfeld, A. (1952) The Principle of Relativity: A Collection of Original Memoirs on the Special and General Theory of Relativity. Courier Corporation.

[5] Klein, M.J. (1961) Max Planck and the Beginnings of the Quantum Theory. Archive for History of Exact Sciences, 1, 459-479. https://doi.org/10.1007/BF00327765

[6] Rovelli, C. (2008) Loop Quantum Gravity. Living Reviews in Relativity, 11, Article No. 5. https://doi.org/10.12942/lrr-2008-5

[7] Lüst, D. and Theisen, S. (1989) Lectures on String Theory. Vol. 346, Springer, Berlin.

[8] Hubble, E. (1929) A Relation between Distance and Radial Velocity among Extra-Galactic Nebulae. Proceedings of the National Academy of Sciences of the United States of America, 15, 168-173. https://doi.org/10.1073/pnas.15.3.168

[9] Caldwell, R.R., Kamionkowski, M. and Weinberg, N.N. (2003) Phantom Energy: Dark Energy with $\mathrm{w}<-1$ Causes a Cosmic Doomsday. Physical Review Letters, 91, 071301. https://doi.org/10.1103/PhysRevLett.91.071301

[10] Trimble, V. (1987) Existence and Nature of Dark Matter in the Universe. Annual Review of Astronomy and Astrophysics, 25, 425-472. https://doi.org/10.1146/annurev.aa.25.090187.002233

[11] Corda, C.H. (2009) Interferometric Detection of Gravitational Waves: The Definitive Test for General Relativity. International Journal of Modern Physics D, 18, 2275-2282. https://doi.org/10.1142/S0218271809015904

[12] Kuo, C.Y., Braatz, J.A., Condon, J.J., Impellizzeri, C.M., Lo, K.Y., Zaw, I. and Greene, J.E. (2010) The Megamaser Cosmology Project. III. Accurate Masses of Seven Supermassive Black Holes in Active Galaxies with Circumnuclear Megamaser Disks. The Astrophysical Journal, 727, 20-34. https://doi.org/10.1088/0004-637X/727/1/20

[13] Erwin, P. and Sparke, L.S. (2003) An Imaging Survey of Early-Type Barred Galaxies. The Astrophysical Journal Supplement Series, 146, 299-352. https://doi.org/10.1086/367885

[14] Greene, J.E., Seth, A., Lyubenova, M., Walsh, J., Van de Ven, G. and Läsker, R. (2014) Circumnuclear Molecular Gas in Megamaser Disk Galaxies NGC 4388 and NGC 1194. The Astrophysical Journal, 788, 145-158. https://doi.org/10.1088/0004-637X/788/2/145

[15] Gilfanov, M. and Merloni, A. (2015) Observational Appearance of Black Holes in X-Ray Binaries and AGN. In: Falanga, M., Belloni, T., Casella, P., Gilfanov, M., Jonker, P. and King, A., Eds., The Physics of Accretion onto Black Holes, Space Sciences Series of ISSI, Springer, New York, 121-148. https://doi.org/10.1007/978-1-4939-2227-7_8

[16] Bouwens, R.J., Thompson, R.I., Illingworth, G.D., Franx, M., Van Dokkum, P.G., Fan, X., Dickinson, M.E., Eisenstein, D.J. and Rieke, M.J. (2004) Galaxies at z 7-8: $\mathrm{z}_{850}$-Dropouts in the Hubble Ultra Deep Field. The Astrophysical Journal, 616, L79-L82. https://doi.org/10.1086/426503

[17] Bouwens, R.J., Illingworth, G.D., Labbe, I., Oesch, P.A., Trenti, M., Carollo, C.M., Van Dokkum, P.G., Franx, M., Stiavelli, M., González, V., Magee, D. and Bradley, L. (2011) A Candidate Redshift $Z \approx 10$ Galaxy and Rapid Changes in That Population at an Age of 500 Myr. Nature, 469, 504-507. https://doi.org/10.1038/nature09717 
[18] Hayes, M., Laporte, N., Pelló, R., Schaerer, D. and Le Borgne, J.-F. (2012) A Peculiar Galaxy Appears at Redshift 11: Properties of a Moderate-Redshift Interloper. Monthly Notices of the Royal Astronomical Society. Letters, 425, L19-L23. https://doi.org/10.1111/j.1745-3933.2012.01293.x

[19] Basu, S. and Das, A. (2019) The Mass Function of Supermassive Black Holes in the Direct-Collapse Scenario. The Astrophysical Journal, 879, L3. .https://doi.org/10.3847/2041-8213/ab2646

[20] Pomarède, D., Courtois, H.M., Hoffman, Y. and Tully, R.B. (2017) Cosmography and Data Visualization. Publications of the Astronomical Society of the Pacific, 129, Article ID: 058002. https://doi.org/10.1088/1538-3873/aa5b73

[21] Amendola, L. and Quercellini, C. (2003) Tracking and Coupled Dark Energy as Seen by the Wilkinson Microwave Anisotropy Probe. Physical Review D, 68, Article ID: 023514. https://doi.org/10.1103/PhysRevD.68.023514

[22] Hui, L., Ostriker, J.P., Tremaine, S. and Witten, E. (2017) Ultralight Scalars as Cosmological Dark Matter. Physical Review D, 95, Article ID: 043541. https://doi.org/10.1103/PhysRevD.95.043541

[23] Schmidt, B.G. (1971) A New Definition of Singular Points in General Relativity. General Relativity and Gravitation, 1, 269-280. https://doi.org/10.1007/BF00759538

[24] Curiel and Erik. (2019) Singularities and Black Holes. Ed., Zalta, E.N., Metaphysics Research Lab, Stanford University.

[25] Bañados, E., Connor, T., Stern, D., Mulchaey, J., Fan, X., Decarli, R., et al. (2018) Chandra X-Rays from the Redshift 7.54 Quasar ULAS J1342+0928. The Astrophysical Journal, 856, L25. https://doi.org/10.3847/2041-8213/aab61e

[26] Volonteri, M., Silk, J. and Dubus, G. (2015) The Case For Supercritical Accretion onto Massive Black Holes at High Redshift. The Astrophysical Journal, 804, 148-155. https://doi.org/10.1088/0004-637X/804/2/148

[27] Kuznetsov, O.A., Lovelace, R.V., Romanova, M.M. and Chechetkin, V.M. (1999) Hydrodynamic Simulations of Counterrotating Accretion Disks. The Astrophysical Journal, 514, 691-703. https://doi.org/10.1086/306964

[28] Shapiro, S.L. and Lightman, A.P. (1976) Black Holes in X-Ray Binaries: Marginal Existence and Rotation Reversals of Accretion Disks. Astrophysical Journal, 204, 555-560. https://doi.org/10.1086/154203

[29] Kaviraj, S., Shabala, S.S., Deller, A.T. and Middelberg, E. (2015) Radio AGN in Spiral Galaxies. Monthly Notices of the Royal Astronomical Society, 454, 1595-1604. https://doi.org/10.1093/mnras/stv1957

[30] Middleton, M.J., Parker, M.L., Reynolds, C.S., Fabian, A.C. and Lohfink, A.M. (2016) The View of AGN-Host Alignment via Reflection Spectroscopy. Monthly Notices of the Royal Astronomical Society, 457, 1568-1576. https://doi.org/10.1093/mnras/stw035

[31] Kinney, W.H. (2003) Cosmology, Inflation, and the Physics of Nothing. In: Prosper, H.B. and Danilov, M., Eds., Techniques and Concepts of High-Energy Physics XII. NATO Science Series (Series II: Mathematics, Physics and Chemistry), Springer, Dordrecht, 189-243. https://doi.org/10.1007/978-94-010-0076-5_5

[32] Seargent, D.A.J. (2015) The Shape, Size, Age and Origin of the Universe. The Shape, Size, Age and Origin of the Universe. In: Weird Universe, Astronomers Universe, Springer, Cham, 113-156. https://doi.org/10.1007/978-3-319-10738-7_3 